\title{
Distributed Diagnosis for Multiprocessor Systems using Extended Local Neighborhoods
}

\author{
Chad C. Lamb \\ XKL \\ 8420 154th Ave NE \\ Redmond, WA 98052
}

\author{
Linda S. DeBrunner \\ Electrical \& Computer Engineering \\ University of Oklahoma \\ Norman, OK 73019
}

\author{
Anindya Das and K. Thulasiraman \\ Computer Science \\ University of Oklahoma \\ Norman, OK 73019
}

\begin{abstract}
We show a solution to the characterization, diagnosability, and diagnosis problems with the $t$-in- $L_{2}$ diagnosability theory for permanently faulty processing elements (PEs). If there are at most $t$ faulty PEs in the local neighborhood set of $x$, and at most $t(m)$ faulty PEs in the extended-local neighborhood set of $x$, then we can uniquely diagnose any graph.
\end{abstract}

\section{INTRODUCTION}

The connectivity of a system is an important measure of fault tolerance. Typical interconnection networks are sparse and symmetrical. The classical diagnosis theories, such as tdiagnosability, are not appropriate for these systems because the number of faulty elements is limited by the minimum node degree, which is very small for sparse systems. Another disadvantage of the classical approach is the need for a supervisory processor. This processor is a bottleneck in the system, unnecessarily burdening the communication paths. The supervisor processor is also a single-point failure in the system. The theory of local diagnosis overcomes these limitations.

In this research, we investigate the local diagnosability of multiprocessor systems for permanently faulty processing elements (PEs). There are three system-level diagnosis problems: the characterization problem is to determine the necessary and sufficient conditions for the diagnosability of a system; the diagnosability problem is to determine the maximum value of certain parameters for which the system is diagnosable; and the diagnosis problem is to find an algorithm that identifies all faulty PEs based on a given syndrome. It is assumed that each set of faulty PEs is from some allowable fault set, which is a restricted set of faults.

We show a solution to the characterization, diagnosability, and diagnosis problems with the $t$-in- $L_{2}$ diagnosability theory. In this theory, if there are at most $t$ faulty PEs in the local neighborhood set of $x$, and at most $t(m)$ faulty PEs in the extended-local neighborhood set of $x$, then we can uniquely diagnose any graph. The extended-local fault constraint, $t(m)$, is a function of the local fault constraint, $t$, and the degree of node $x$. We develop a general approach for the tin- $\mathrm{L}_{2}$ diagnosis of an arbitrary graph.

\section{II.BACKGROUND}

The connectivity of a system is an important measure of fault tolerance. Typical interconnection networks are sparse and symmetrical, like the mesh and hypercube. The classical diagnosis theories, such as t-diagnosability, are not appropriate for these systems because the number of faulty PEs is limited by the minimum degree of all PEs in the system, which is very small for sparse systems. Another disadvantage of the classical approach is the need for a supervisory PE. This PE is a bottleneck in the system, a single-point failure, unnecessarily burdening the communication paths of the system. The theory of local diagnosis overcomes these limitations.

A classic paper by Preparata, Metze, and Chien was written over thirty years ago on system-level fault diagnosis [1]. In this paper, the authors describe a general procedure to identify faulty and fault-free PEs in a multiprocessor system. Each PE is tested by other PEs in the system. The authors make no assumptions about the PEs, i.e., each PE can be either faulty or fault-free. However, the specific model used to determine the test results and the interpretation of those test results is not specified.

There are three popular models in the literature [2] for the interpretation of the test results: the symmetric invalidation model (PMC model) [1], asymmetric invalidation model (BGM model) [3], and the comparison model [2][4]. In the PMC model, the result of a test is reliable only if the testing $\mathrm{PE}$ is fault-free. If a faulty $\mathrm{PE}$ is testing another $\mathrm{PE}$ in the system, the result will be unreliable. An unreliable test result is shown as a $1 \backslash 0$. The syndrome must be interpreted to determine the faulty PEs. In the BGM model, when a faulty $\mathrm{PE}$ tests another faulty PE, the test outcome will be a 1 . So, unlike the PMC model, the testing PE can be faulty, and the test result will be reliable if the other $P E$ is also faulty. If a faulty PE tests a fault-free PE, the test outcome is unreliable.

In the PMC and BGM models, a PE will test another PE (or PEs) in the system. The test links are uni-directional. In the comparison model, the test links are bi-directional. To implement this, the same task is assigned to every PE in the system, and the outputs are compared. A test outcome (comparison) is unreliable only if both PEs are faulty. Similar to the other two models, the syndrome must be interpreted to determine which nodes are actually faulty.

In this research, we investigate local diagnosis approaches to system-level diagnosis using the comparison model. Each node uses information from its immediate neighbors and from their neighbors. We refer to this as the local neighborhood and the extended-local neighborhood.

\section{Local DIAGNOSIS}

Determining if a system is locally diagnosable is more practical than the global diagnosis methods. One advantage is that nodes in the system can be tested simultaneously. This can reduce test time dramatically. Also, the fault constraints for global diagnosis are impractical, since there are relatively few allowable faults compared to the number of PEs in a multiprocessor system. For example, a 1024 node system configured as a mesh is only 3-diagnosable in the traditional global diagnosis approach, since it can tolerate at most 1 less than the connectivity of the entire system. The local 
diagnosis approach for the same 1024 node system can tolerate 511 faults.

In [5], the $\mathrm{t}$-in- $\mathrm{L}_{1}$ diagnosis theory is developed. In this theory, a graph $\mathrm{G}(U, \mathrm{E})$ is defined to be $\mathrm{t}$-in- $\mathrm{L}_{1}$ diagnosable if all faulty nodes can be uniquely diagnosed, assuming that there are fewer than $\mid U / 2$ faulty nodes in $\mathrm{G}$, and there are no more than $\left\{\frac{d(x)}{2}\right]+1$ faults in the local neighborhood of $x$, $\forall \mathrm{x} \in U$. For this research, we develop a new approach to local diagnosis: the $\mathrm{t}$-in- $\mathrm{L}_{2}$ diagnosis theory. We first define our notation.

Let the nodes in $L(x)$ be identified as the set $\left\{z_{1}, z_{2}, \ldots\right.$, $\left.z_{d(x)}\right\}$ and the nodes in $L\left(z_{i}\right)$ be identified as the set $\left\{x, z_{i 1}, z_{i 2}\right.$, $\left.\ldots, z_{i(d(x)-1)}\right\}$, where $i=1,2, \ldots, d(x)$. This is shown Figure 1 for a regular graph of degree 4 . We call the set $L(x) \cup\{x\}$, the local set of $x$, and the set $\mathrm{L}(\mathrm{L}(\mathrm{x})) \cup \mathrm{L}(\mathrm{x}) \cup\{\mathrm{x}\}$, the extendedlocal set of $x$ (we have simplified the notation by letting $\mathrm{L}(\{\mathrm{L}(\mathrm{x})\})=\mathrm{L}(\mathrm{L}(\mathrm{x}))$, which represents the set of nodes that are distance 2 from $x$ ). The local set of $x$ and extended-local set of $x$ are shown by the shaded nodes in Figure 2a and Figure $2 b$, respectively.

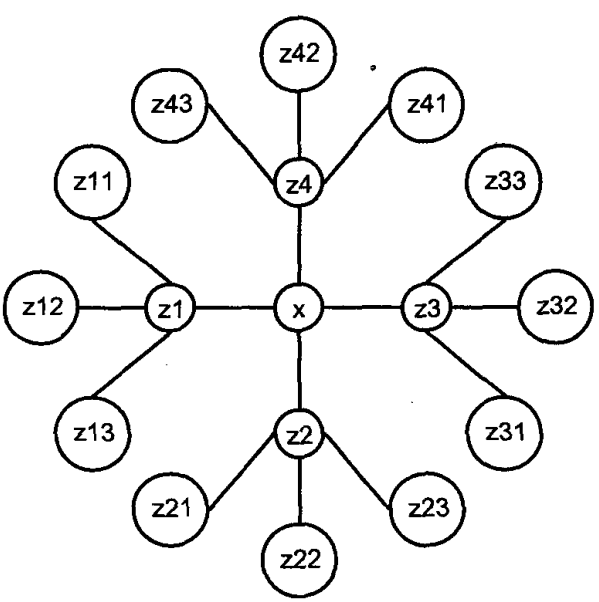

Figure 1. Regular graph with minimum cycle length 5
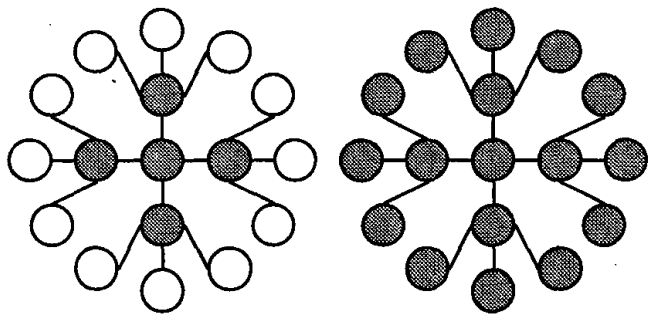

Local Set of $x$

Extended-Local Set of $x$ (a)

(b)

Figure 2. Local and Extended-Local Set of $x$
Let $\bar{N}(\{x\})$ be the set of nodes in $L(x)$ that share a 0 -link with $x$ and $N(\{x\})$ be the set of nodes in $L(x)$ that share a 1link with $x$. To simplify the notation, we call $\bar{N}(\{x\}) \rightarrow \bar{N}$ and $N(\{x\}) \rightarrow N$. Similarly, $\bar{N}(\bar{N})$ is the set of nodes that share a 0 -link with the nodes in $\bar{N}$ (not including the nodes in $\bar{N}), \bar{N}(\mathbb{N})$ is the set of nodes that share a 0 -link with the nodes in $\mathrm{N}$ (not including the nodes in $\mathrm{N}_{2} \mathrm{~N}(\bar{N})$ is the set of nodes that share a 1-link with the nodes $N$ (not including the nodes in $\bar{N}$ ), and $N(N)$ is the set of nodes that share a 1-link with the nodes in $\mathrm{N}$ (not including the nodes in $\mathrm{N}$ ).

Let $N_{i}$ be the node $z_{i} \in N$ that shares a 1-link with $x$ and $\mathrm{N}\left(\mathrm{N}_{\mathrm{i}}\right)$ be the set of nodes that share a 1-link with $\mathrm{z}_{\mathrm{i}}$, not including the node $z_{i}$. Similarly $N\left(N_{i}\right)$ is the set of nodes that share a 0 -link with $z_{i}$. Referring to Figure $3, N=\left\{z_{2}\right.$, $\left.\mathrm{z}_{3}\right\}, \overline{\mathbf{N}}=\left\{\mathrm{z}_{1}, \mathrm{z}_{4}\right\}, \overline{\mathbf{N}}(\overline{\mathbf{N}})=\left\{\mathrm{z}_{42}\right\}, \overline{\mathbf{N}}(\mathbf{N})=\left\{\mathrm{z}_{22}, \mathrm{z}_{32}\right\}, \mathbf{N}(\mathbf{N})$ $=\left\{z_{11}, z_{12}, z_{13}, z_{41}, z_{43}\right\}, N(N)=\left\{z_{21}, z_{23}, z_{31}, z_{33}\right\}, N\left(N_{i}\right)=\{$ $\left.z_{11}, z_{12}, z_{13}\right\}$.

\section{II. $\quad \mathrm{t}$-in- $\mathrm{L}_{2}$ DIAGNOSIS}

In the following arguments, the local fault constraint (lfc) is the number of faulty nodes in the local set of $x$ that are allowed. The extended-local fault constraint (elfc) is the number of faulty nodes in the extended-local set of $x$ that are allowed.

\section{Lemma 1}

Given a graph $\mathrm{G}$ with a local fault constraint (lfc) of no more than $\mathrm{t}$ faults in $\mathrm{L}(\mathrm{x}) \cup\{\mathrm{x}\}, \forall \mathrm{x} \in U$, where $\mathrm{t}=\mathrm{d}(\mathrm{x})-1$ and $\mathrm{F}$ is the set of faulty nodes in $G$, then

1. If $|N|>t$, then $x \in F$

2. If $|\mathrm{N}|<\mathrm{d}(\mathrm{x})-\mathrm{t}+1$, then $\mathrm{x} \in U-\mathrm{F}$.

\section{Lemma 2}

Given a graph $\mathrm{G}$, with a local fault constraint (lfc) of no more than $\mathrm{t}_{\mathrm{x}}$ faults in $\mathrm{L}(\mathrm{x}) \cup\{\mathrm{x}\}, \forall \mathrm{x} \in U$, and no more than $\mathrm{t}_{\mathrm{i}}$ faults in $\mathrm{L}\left(\mathrm{z}_{\mathrm{i}}\right) \cup\left\{\mathrm{z}_{\mathrm{i}}\right\}, \forall \mathrm{z}_{\mathrm{i}} \in \mathrm{L}(\mathrm{x})$, where $\mathrm{t}_{\mathrm{x}} \leq \mathrm{d}(\mathrm{x})-1$ and $\mathrm{t}_{\mathrm{i}} \leq \mathrm{d}\left(\mathrm{z}_{\mathrm{i}}\right)-1$. Let $F$ be the set of faulty nodes in $G$, then

1. If $|N| \leq d(x)-t_{x}+1$ and $\exists z_{i}$, such that $\left|N\left(N_{i}\right)\right| \geq t_{i}$, then $x \in U-F$.

2. If $d(x)-t_{x}+2 \leq|N| \leq t_{x}$ and $\left|N\left(N_{i}\right)\right| \geq t_{i}$, for $i=1$ to [ $[N \mid-$ $\left.\left(d(x)-t_{x}\right)\right]$, then $x \in U-F$.

3. If $\exists z_{i}$, such that $\left|N\left(N_{i}\right)\right|<\left[d\left(z_{i}\right)-t_{i}\right]$, then $x \in F$.

Lemma 3

Given a graph $\mathrm{G}$, and syndrome $\mathrm{S}$, let $\mathrm{F}$ be an allowable fault set for $\mathrm{S}$ such that $\mathrm{F} \neq U$. Let $\mathrm{C}$ (or $\mathrm{C}^{\prime}$ ) be the set of nodes that have multiple paths of length 2 with $\mathrm{x}$ and are counted more than once in the computation of $F(x)$ (or $F^{\prime}(x)$ ). Then, the number of faulty nodes in the extended-local set of $x$, $\forall \mathrm{x} \in U$ is given by:

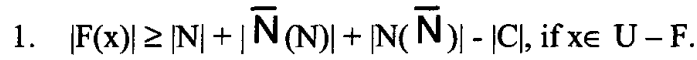

2. $\left|F^{\prime}(x)\right| \geq 1+|\bar{N}|+|\bar{N}(\bar{N})|+$

$$
\left.\left.\sum_{i=1}^{|N|} \min \| N\left(N_{i}\right)\left|, d\left(Z_{i}\right)-\right| N\left(N_{i}\right)\right]\right]-\left|C^{\prime}\right|, \text { if } x \in F \text {. }
$$




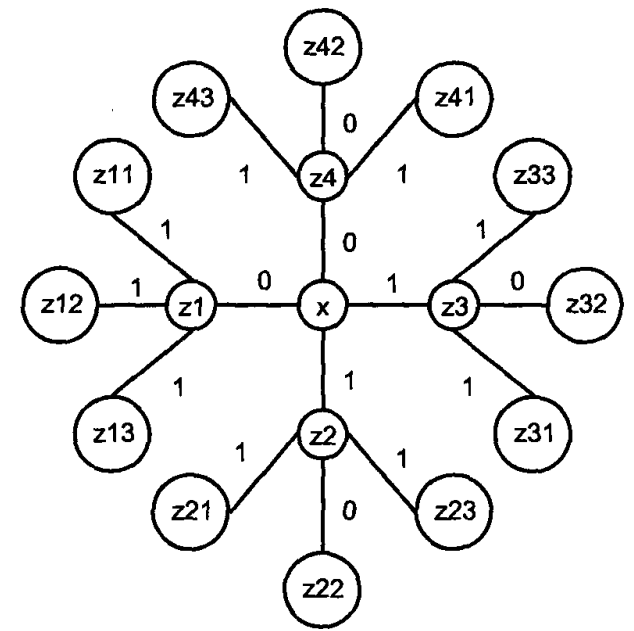

Figure 3. Graph with a syndrome

Theorem 1

Given a graph $\mathrm{G}$, and syndrome $\mathrm{S}$, let $\mathrm{F}$ be an allowable fault set for $S$ such that $F \neq U$. If there are no more than $t_{x}=d(x)$ $\mathrm{m}$ faults in $\mathrm{L}(\mathrm{x}) \cup\{\mathrm{x}\}, \forall \mathrm{x} \in U$, where $1 \leq \mathrm{m} \leq \frac{\mathrm{d}(\mathrm{x})}{2}-1$, and no more than $t(m)$ (from table 1) faults in $\mathrm{L}(\mathrm{L}(\mathrm{x})) \cup \mathrm{L}(\mathrm{x}) \cup\{\mathrm{x}\}$, $\forall \mathrm{x} \in U$, then $\mathrm{G}$ is $\mathrm{t}$-in- $\mathrm{L}_{2}$ diagnosable.

\section{Proof Outline:}

To prove the $t-i n-L_{2}$ diagnosability of a graph $G$, we must show that if the local and extended-local fault constraints are not violated, then the fault set $F$ is unique for any given syndrome. We show that $\mathrm{x} \in \mathrm{F}$ if and only if at least one of the following conditions are met:

1. $|\mathbf{N}|>\mathrm{t}_{\mathrm{x}}$

2. $\exists z_{i}$, such that $\left|N\left(N_{i}\right)\right|<\left[d\left(z_{i}\right)-t_{i}\right]$

3. $|F(x)|>t(m)$, where $F(x)=N \cup \bar{N}(N) \cup N(\bar{N})$.

\section{CONCLUSIONS}

We have shown a new diagnosability criterion in this research, $t$-in- $\mathrm{L}_{2}$ diagnosis. This theory is applied to regular structures that are distributed and sparse, with examples shown for the mesh, hypercube, and hexagon structures. We improved on existing algorithms in local diagnosis [5][6], while generalizing the applicable structures. We eliminated the constraints imposed by those local diagnosis approaches, while allowing more faults in the system.

TABLE I

$t$ AND $t(m)$ FOR THE GENERAL REGULAR GRAPH, MESH, AND HYPERCUBE

\begin{tabular}{|c|c|c|c|c|c|}
\hline $\mathbf{m}$ & $\begin{array}{c}\quad l f c \\
t_{x}=d(x)-m \\
t_{i}=d\left(z_{j}\right)-m\end{array}$ & $\begin{array}{c}\text { general } \\
\text { regular gra } \\
t(\mathbf{m})\end{array}$ & & $\begin{array}{l}\text { mesh } \\
\mathrm{t}(\mathrm{m})\end{array}$ & $\begin{array}{c}\text { hypercube } \\
t(m)\end{array}$ \\
\hline 1 & $d(x)-1$ & $\left\lfloor\frac{3 \cdot d(x)}{2}\right\rfloor$ & +1 & 6 & n \\
\hline 2 & $\mathrm{~d}(\mathrm{x})-2$ & $\left\lfloor\frac{4 \cdot d(x)}{2}\right\rfloor$ & +4 & $\mathrm{~N} / \mathrm{A}$ & $\frac{5 \cdot n}{4}+2$ \\
\hline 3 & $d(x)-3$ & $\left\lfloor\frac{5 \cdot d(x)}{2}\right\rfloor$ & +9 & N/A & $\frac{6 \cdot n}{4}+4$ \\
\hline . & . & . & & . &. \\
\hline . & : & $\cdot$ & &. & $\therefore$ \\
\hline $\mathrm{m}$ & $d(x)-m$ & $\frac{(2+m) \cdot d(x}{2}$ & $\underline{-}\rfloor+m^{2}$ & $6, m=1$ & $\frac{(3+m) \cdot n}{4}+\left\lfloor\frac{m^{2}}{2}\right.$ \\
\hline $\mathrm{m}=\frac{\mathrm{d}(\mathrm{x})}{2}-1$ & $\frac{d(x)}{2}+1$ & $\frac{\mathrm{d}(\mathrm{x})^{2}-\mathrm{d}(\mathrm{x}}{2}$ & +1 & N/A & $\frac{\mathrm{n}^{2}}{4}$ \\
\hline
\end{tabular}


In the $\mathrm{t}$-in- $\mathrm{L}_{2}$ diagnosis theory, we require no more than $\mathrm{t} \leq$ $d(x)-m$ faults in $L(x) \cup\{x\}$ and no more than $t(m)=$ $\left\lfloor\frac{(2+\mathrm{m}) \cdot \mathrm{d}(\mathrm{x})}{2}\right\rfloor+\mathrm{m}^{2}$ fault in $\mathrm{L}(\mathrm{L}(\mathrm{x})) \cup \mathrm{L}(\mathrm{x}) \cup\{\mathrm{x}\}, \forall \mathrm{x} \in$ $U$, for a regular graph with minimum cycle length of 5 (and $t(m)=\frac{(3+m) \cdot n}{4}+\left\lfloor\frac{m^{2}}{2}\right\rfloor$ for a hypercube). We noted that the local fault constraint for the $\mathrm{t}$-in- $\mathrm{L}_{1}$ diagnosis theory is the same as the local fault constraint for the $t-i n-L_{2}$ diagnosis theory when $\mathrm{m}=\frac{\mathrm{d}(\mathrm{x})}{2}-1$, which is the lower bound on $\mathrm{m}$. We know that $\mathrm{t} \geq \frac{\mathrm{d}(\mathrm{x})}{2}+1$ in the $\mathrm{t}-\mathrm{in}-\mathrm{L}_{2}$ diagnosability theory (which corresponds to $\mathrm{m} \leq \frac{\mathrm{d}(\mathrm{x})}{2}-1$ ). This value for $t$ is consistent with a majority voting scheme. In a majority voting scheme, $t \leq\left\lfloor\frac{d(x)}{2}\right\rfloor$ so that the majority of the nodes in $L(x)$ cannot be faulty, which shows that if $t \leq$ $\left\lfloor\frac{d(x)}{2}\right\rfloor$ then we do not need an extended-local fault constraint $t(m)$.

The local fault constraint $\mathrm{t}$ can be as large as $\mathrm{d}(\mathrm{x})-1$, thereby allowing for a very large number of faults in $\mathrm{L}(\mathrm{x}) \cup\{\mathrm{x}\}, \forall \mathrm{x} \in U$. This has not been done in any other deterministic approach. In the t-diagnosis approach, for example, there can be $d(x)-1$ faults in $L(x) \cup\{x\}$, for only one such $\mathrm{x} \in U$, and for the $\mathrm{t}$-in- $\mathrm{L}_{1}$ approach there can be at most $\left\lfloor\frac{\mathrm{d}(\mathrm{x})}{2}\right\rfloor+1$ faults in $\mathrm{L}(\mathrm{x}) \cup\{\mathrm{x}\}, \forall \mathrm{x} \in U$

\section{REFERENCES}

[1] F. P. Preparata, G. Matze, R. T. Chien, "On the Connection Assignment Problem of Diagnosable Systems," IEEE Trans. Electr. Compt., Vol. EC-16, pp. 848-854, 1967.

[2] A. K. Somani, V. K. Agarwal, D. Avis, "A Generalized Theory for System Level Diagnosis," IEEE Trans. on Computers, vol. C-36, pp. 538-546, May 1987.

[3] F. Barsi, Grandoni, P. Maestrini, "A Theory of Diagnosability of Digital Systems," IEEE Trans. on Computers, vol. C-25, pp. 585-593, 1976.

[4] M. Malek, "A Comparison Connection Assignment for Diagnosis of Multiprocessor Systems," Proc. 17th International Symposium on Computer Architecture, pp. 31-35, 1980.

[5] A. Das, K. Thulasiraman, V. K. Agarwal, K. B. Lakshmanan, "Multiprocessor Fault Diagnosis Under Local Constraints," IEEE Trans. on Computers, vol. 42, pp. 984-988, Aug. 1993.

[6] A. K. Somani and V. K. Agarwal, "Distributed Diagnosis Algorithms for Regular Interconnected Structures," IEEE Trans. on Computers, vol. 41, pp. 899-906, July 1992. 